\title{
Mediterranean diet as the ideal model for preventing non-alcoholic fatty liver disease (NAFLD)
}

\author{
Miguel A. Martínez-González ${ }^{1,2,3}$, Gorka Bastarrika ${ }^{4}$ \\ ${ }^{1}$ Centro de Investigación Biomédica en Red Fisiopatología de la Obesidad y la Nutrición (CIBEROBN), Institute of Health Carlos III, Madrid, \\ Spain; ${ }^{2}$ University of Navarra, Department of Preventive Medicine and Public Health, IDISNA, Pamplona, Spain; ${ }^{3}$ Department of Nutrition, \\ Harvard T. H. Chan School of Public Health, Boston, MA, USA; ${ }^{4}$ University of Navarra, Department of Radiology, Clínica Universidad de Navarra, \\ Pamplona, Spain \\ Correspondence to: Prof. Miguel A. Martínez-González, MD, MPH, PhD. University of Navarra, Department of Preventive Medicine and Public \\ Health, C/Irunlarrea, 31008 Pamplona, Spain. Email: mamartinez@unav.es. \\ Provenance and Peer Review: This article was commissioned by the editorial office, Hepatobiliary Surgery and Nutrition. The article did not undergo \\ external peer review.
}

Submitted Oct 24, 2019. Accepted for publication Nov 05, 2019.

doi: $10.21037 /$ hbsn.2019.11.13

View this article at: http://dx.doi.org/10.21037/hbsn.2019.11.13

The prevalence of nonalcoholic fatty liver disease (NAFLD) has dramatically increased in the 2-3 last decades and it represents the most frequent global cause of liver disease, affecting $25 \%$ to $45 \%$ of adults in most studies (1). The worldwide elevation in the population rates of NAFLD has come in parallel with rising unprecedented pandemics of obesity and diabetes. There is no specific medication for NAFLD and dietary/lifestyle modifications are the main foundations for the treatment of NAFLD. They are also very likely to be effective for its primary prevention. In this context, a dietary pattern that meets most requirements to become the ideal model for the prevention of NAFLD is the traditional Mediterranean diet (2). In addition to the potential for preventing NAFLD, the Mediterranean diet has sufficiently demonstrated its great ability to improve cardiovascular health. The accrual of prospective and well conducted studies showing cardiovascular benefits associated with better adherence to a high-quality food pattern is superior for the traditional Mediterranean food pattern than for any other dietary pattern (3). Also, only for the Mediterranean diet, secondary prevention randomized trials $(4,5)$ and a large primary prevention trial (6) have demonstrated substantial benefits. This large primary prevention trial is the Spanish PREDIMED trial $(6,7)$, that included 7,447 initially healthy participants $(42.5 \%$ of them were men aged $55-80$ years and $57.5 \%$ were women aged $60-80$ years). All participants were at high risk of cardiovascular disease and they were randomized to 3 equally sized groups to receive 3 different dietary interventions: (I) a Mediterranean Diet supplemented with extra-virgin olive oil (MedDiet + EVOO); (II) a Mediterranean diet supplemented with mixed nuts (MedDiet + nuts); or (III) a control diet (low-fat diet).

In a subset of 100 participants from one of the 11 centers of the PREDIMED trial, Pintó et al. recently evaluated the role of the dietary intervention on the occurrence of NAFLD as determined by magnetic resonance imaging (MRI) (8). They found that, after a median follow-up of $3 y$, the prevalence of NAFLD and the average fat liver content were significantly lower in the MedDiet + EVOO than in the control group. Despite the limitation of not collecting baseline measurements of liver fat, this is an important finding. It should be put in the context of previous observational studies and randomized trials that also reported benefits for the Mediterranean diet as compared to a low-fat diet.

Another important trial is the CENTRAL 18-month intervention trial, where a Mediterranean/low-carbohydrate diet also achieved a greater decrease in hepatic fat content than a low-fat diet (9). In the CENTRAL trial, $53 \%$ of participants had NAFLD at baseline, but at 18-month follow-up, the beneficial effect of the Mediterranean/low carbohydrate diet on liver fat reduction versus the lowfat diet was significant even in patients without NAFLD 
at baseline (9). Previous studies assessing the role of Mediterranean dietary pattern in patients with NAFLD exhibited several limitations, including the use of crosssectional designs with inherent limitations for drawing causal relationships, only longitudinal observational studies with small sample sizes and only short-term follow-up, and, above all, a suboptimal approach for the evaluation of the outcome (10).

Considerable strengths of both the PREDIMED and the CENTRAL trial is that they assessed an intervention following a randomized design and they used MRI to assess liver fat content after a sufficiently long follow-up period.

Traditionally, liver biopsy has been performed to determine the presence and severity of NAFLD. Liver biopsy has also been the classical approach in clinical as well as in research settings for selection of patients for treatment or for the development of new drugs. Furthermore, patients may undergo repeated liver biopsies to monitor disease progression. Liver biopsy, however, is invasive in nature and possesses a small but not negligible risk of complications, such as bleeding. Moreover, because a biopsy specimen reflects a very small sample of the liver, underestimation of disease is not uncommon, and quantification generally remains subjective. Therefore, there is increased interest in developing noninvasive assessment approaches that may help in diagnosis and reliable, reproducible and repeatable quantification of NAFLD. Among those, particularly MRI is gaining relevance. In both the study by Pintó et al. (8) and the study by Gepner et al. (9), the authors used MRI to determine the percentage of liver fat and diagnosis of steatosis.

Magnetic resonance spectroscopy (MRS) is considered the non-invasive reference standard to classify presence and amount of liver fat. However, availability of MRS is limited and cannot be routinely performed. New MRI methods based on proton density fat fraction (PDFF) quantification have been developed (10). Recent studies demonstrate that liver fat content estimated by MRI- PDFF correlates well with that measured by MRS (11) and with histology $(11,12)$. Also, MRI- PDFF methods seem to be more sensitive than histology in quantifying changes in the liver fat content over time and also allow for follow-up of responses after intervention (13). Interestingly, MRI-PDFF estimates are now available in clinical scanners, so that MRI- PDFF maps can be generated inline and can be easily analyzed after minimal training.

\section{Acknowledgments}

Funding: None.

\section{Footnote}

Conflicts of Interest: Both authors have completed the ICMJE uniform disclosure form (available at http://dx.doi. org/10.21037/hbsn.2019.11.13). The authors have no conflicts of interest to declare.

Ethical Statement: The authors are accountable for all aspects of the work in ensuring that questions related to the accuracy or integrity of any part of the work are appropriately investigated and resolved.

Open Access Statement: This is an Open Access article distributed in accordance with the Creative Commons Attribution-NonCommercial-NoDerivs 4.0 International License (CC BY-NC-ND 4.0), which permits the noncommercial replication and distribution of the article with the strict proviso that no changes or edits are made and the original work is properly cited (including links to both the formal publication through the relevant DOI and the license). See: https://creativecommons.org/licenses/by-nc$\mathrm{nd} / 4.0 \%$.

\section{References}

1. Rinella ME. Nonalcoholic fatty liver disease: a systematic review. JAMA 2015;313:2263-73.

2. Ma J, Hennein R, Liu C, et al. Improved Diet Quality Associates With Reduction in Liver Fat, Particularly in Individuals With High Genetic Risk Scores for Nonalcoholic Fatty Liver Disease. Gastroenterology 2018;155:107-17.

3. Martínez-González MA, Gea A, Ruiz-Canela M. The Mediterranean Diet and Cardiovascular Health. Circ Res 2019;124:779-98.

4. de Lorgeril M, Salen P, Martin JL, et al. Mediterranean diet, traditional risk factors, and the rate of cardiovascular complications after myocardial infarction: final report of the Lyon Diet Heart Study. Circulation 1999;99:779-85.

5. Giannuzzi P, Temporelli PL, Marchioli R, et al. Global secondary prevention strategies to limit event recurrence 
after myocardial infarction: results of the GOSPEL study, a multicenter, randomized controlled trial from the Italian Cardiac Rehabilitation Network. Arch Intern Med 2008;168:2194-204.

6. Estruch R, Ros E, Salas-Salvadó J, et al. Primary Prevention of Cardiovascular Disease with a Mediterranean Diet Supplemented with Extra-Virgin Olive Oil or Nuts. N Engl J Med 2018;378:e34.

7. Martínez-González MA, Salas-Salvadó J, Estruch R, et al. Benefits of the Mediterranean Diet: Insights From the PREDIMED Study. Prog Cardiovasc Dis 2015;58:50-60.

8. Pintó X, Fanlo-Maresma M, Corbella E, et al. A Mediterranean Diet Rich in Extra-Virgin Olive Oil Is Associated with a Reduced Prevalence of Nonalcoholic Fatty Liver Disease in Older Individuals at High Cardiovascular Risk. J Nutr 2019;149:1920-9.

9. Gepner Y, Shelef I, Komy O, et al. The beneficial effects of Mediterranean diet over low-fat diet may be

Cite this article as: Martínez-González MA, Bastarrika G. Mediterranean diet as the ideal model for preventing nonalcoholic fatty liver disease (NAFLD). HepatoBiliary Surg Nutr 2020;9(3):379-381. doi:10.21037/hbsn.2019.11.13 mediated by decreasing hepatic fat content. J Hepatol 2019;71:379-88.

10. Anania C, Perla FM, Olivero F, et al. Mediterranean diet and nonalcoholic fatty liver disease. World J Gastroenterol 2018;24:2083-94.

11. Idilman IS, Keskin O, Celik A, et al. A comparison of liver fat content as determined by magnetic resonance imaging-proton density fat fraction and MRS versus liver histology in non-alcoholic fatty liver disease. Acta Radiol 2016;57:271-8.

12. Yokoo T, Serai SD, Pirasteh A, et al. Linearity, Bias, and Precision of Hepatic Proton Density Fat Fraction Measurements by Using MR Imaging: A Meta-Analysis. Radiology 2018;286:486-98.

13. Noureddin M, Lam J, Peterson MR, et al. Utility of magnetic resonance imaging versus histology for quantifying changes in liver fat in nonalcoholic fatty liver disease trials. Hepatology 2013;58:1930-40. 